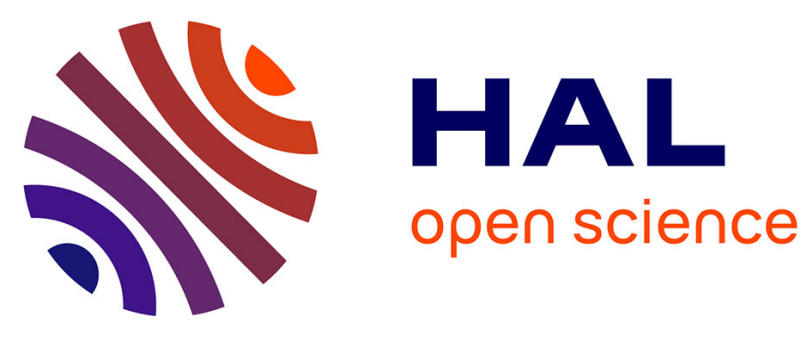

\title{
Multilevel systems biology modeling characterized the atheroprotective efficiencies of modified dairy fats in a hamster model
}

Jean-Charles Martin, Amélie Berton, Christian Ginies, Romain Bott, Pierre Scheercousse, Alessandra Saddi, Daniel Gripois, Jean-Francois Landrier, Daniel Dalemans, Marie-Christine Alessi, et al.

\section{To cite this version:}

Jean-Charles Martin, Amélie Berton, Christian Ginies, Romain Bott, Pierre Scheercousse, et al.. Multilevel systems biology modeling characterized the atheroprotective efficiencies of modified dairy fats in a hamster model. AJP - Heart and Circulatory Physiology, 2015, 309 (5), pp.H935-H945. 10.1152/ajpheart.00032.2015 . hal-02639610

\section{HAL Id: hal-02639610 \\ https://hal.inrae.fr/hal-02639610}

Submitted on 28 May 2020

HAL is a multi-disciplinary open access archive for the deposit and dissemination of scientific research documents, whether they are published or not. The documents may come from teaching and research institutions in France or abroad, or from public or private research centers.
L'archive ouverte pluridisciplinaire HAL, est destinée au dépôt et à la diffusion de documents scientifiques de niveau recherche, publiés ou non, émanant des établissements d'enseignement et de recherche français ou étrangers, des laboratoires publics ou privés.

$$
\text { Copyright }
$$




\title{
Multilevel systems biology modeling characterized the atheroprotective efficiencies of modified dairy fats in a hamster model
}

\author{
Jean-Charles Martin, ${ }^{1}$ Amélie Berton, ${ }^{1}$ Christian Ginies, ${ }^{1}$ Romain Bott, ${ }^{1}$ Pierre Scheercousse, ${ }^{1}$ \\ Alessandra Saddi, ${ }^{1}$ Daniel Gripois, ${ }^{2}$ Jean-François Landrier, ${ }^{1}$ Daniel Dalemans, ${ }^{3}$ Marie-Christine Alessi, ${ }^{1}$ \\ and Bernadette Delplanque ${ }^{2}$ \\ ${ }^{1}$ INRA UMR1260, Nutrition, Obésité et Risque Thrombotique, Marseille, France; Aix-Marseille Université, Faculté de \\ Médecine, Marseille, France; and INSERM, UMR1062, Nutrition, Obésité et Risque Thrombotique, Marseille, France; ${ }^{2}$ UMR \\ 8195 Centre de Neurosciences Paris-Sud, Neuroendocrinologie Moléculaire de la Prise Alimentaire, Université Paris-Sud XI, \\ Orsay, France; and ${ }^{3}$ Corman, S.A., Goé, Belgium
}

Submitted 15 January 2015; accepted in final form 15 June 2015

Martin JC, Berton A, Ginies C, Bott R, Scheercousse P, Saddi A, Gripois D, Landrier JF, Dalemans D, Alessi MC, Delplanque B. Multilevel systems biology modeling characterized the atheroprotective efficiencies of modified dairy fats in a hamster model. Am J Physiol Heart Circ Physiol 309: H935-H945, 2015. First published June 19, 2015; doi:10.1152/ajpheart.00032.2015.-We assessed the atheroprotective efficiency of modified dairy fats in hyperlipidemic hamsters. A systems biology approach was implemented to reveal and quantify the dietary fat-related components of the disease. Three modified dairy fats (40\% energy) were prepared from regular butter by mixing with a plant oil mixture, by removing cholesterol alone, or by removing cholesterol in combination with reducing saturated fatty acids. A plant oil mixture and a regular butter were used as control diets. The atherosclerosis severity (aortic cholesteryl-ester level) was higher in the regular butter-fed hamsters than in the other four groups $(P<0.05)$. Eighty-seven of the 1,666 variables measured from multiplatform analysis were found to be strongly associated with the disease. When aggregated into 10 biological clusters combined into a multivariate predictive equation, these 87 variables explained $81 \%$ of the disease variability. The biological cluster "regulation of lipid transport and metabolism" appeared central to atherogenic development relative to diets. The "vitamin E metabolism" cluster was the main driver of atheroprotection with the best performing transformed dairy fat. Under conditions that promote atherosclerosis, the impact of dairy fats on atherogenesis could be greatly ameliorated by technological modifications. Our modeling approach allowed for identifying and quantifying the contribution of complex factors to atherogenic development in each dietary setup.

atherosclerosis; dairy fats; systems biology; metabolomics; cholesterol

\section{NEW \& NOTEWORTHY}

A technologically modified dairy fat, combining decreased saturated fatty acids, low cholesterol, and both high in rumenic acid and $\gamma$-tocopherol, was highly potent in reducing atherogenesis in a diet-induced atherogenic hamster model. The biological impact of the diets on the atherogenic outcomes was revealed by a new systems biology modeling.

THE CONSUMPTION OF SATURATED fat has been frequently associated with an increase in LDL-cholesterol (67), which is a major cardiovascular risk factor, and with coronary heart diseases

Address for reprint requests and other correspondence: J.-C. Martin, UMR INRA1260/INSERM1060/AMU, Nutrition, Obésité et Risque Thrombotique, 27 bvd Jean Moulin, Fac Méd La Timone, F-13385 Marseille, France (e-mail: jean-charles.martin@univ-amu.fr).
(19). Although less thoroughly documented, cholesterol intake in "high absorber" individuals has also been found to increase LDL-cholesterol levels (65). Because the consumption of dairy fats contributes to the dietary intake of these lipids, physicians recommend reducing the consumption of dairy products by at-risk individuals $(47,62)$. However, this point of view has been challenged by numerous clinical and epidemiological studies (reviewed in Ref. 14, 20, 37, 42), many of which have noted that dairy fats also increase the beneficial HDL-cholesterol level, which could counterbalance the increase in LDLcholesterol levels. Dairy fats also contain short to medium saturated fatty acids, which have not been associated with coronary heart diseases (19). Additionally, simply banning dairy foods would be inappropriate because their consumption has also been connected to health benefits (30). The latest recommendations could reconcile this apparent contradiction by reporting that some caution should be taken only in conditions of high overall saturated fat intake (more than $12 \%$ of total energy intake) (29). However, simply relying on nutritional guidelines for recommendations is not sufficient because the translation of guidelines into real-life situations can be weak (61). Hence, a beneficial strategy would be to modify dairy fats to help individuals comply with recommendations (30) without losing the health benefits of dairy products by eliminating them altogether.

In fact, several strategies have aimed to manipulate the composition of dairy fats to improve their cardiovascular features further $(21,30,41,45,51)$. Targeting both saturated fatty acids and cholesterol in milk fat has appeared advantageous in humans with regard to cardiovascular disease risk (21, $41,51)$. To target these components, zootechnical means, such as modifying the cattle diet by providing polyunsaturated fatty acids, have been used $(41,51)$, but there is a physiological limitation on the extent to which the profiles of fatty acids can be modified, and the cholesterol content cannot be changed. Physicochemical techniques allow for more important modifications, such as physical fractionation, interesterification, mixing with other fats, and the removal of cholesterol.

We thus designed a study that examined the atherosclerotic impacts of various technologically transformed dairy fats in a hamster model that we and others have found suitable for addressing this issue $(23,33,69)$. The occurrence of atherosclerosis, which constitutes one of the main pathological foundations of cardiovascular disease, is highly multifactorial. Hence, evaluating and comparing dietary strategies to improve atherosclerosis also require the examination of a comprehen- 
sive set of disease components in addition to measuring the severity of vascular lesions (34). We thus designed a systems biology approach for identifying the diet-related components of the disease and assessing how they could be differentially modified by each dietary treatment. To achieve these goals, we implemented a multiplatform analysis combining conventional blood clinical chemistry and fatty acid analyses, broad atherosclerosis-related gene expression analysis of blood and liver samples, and untargeted metabolite profiling of biofluids. The complex biological information obtained was condensed into a reduced number of biological sets, which were modeled into scores to facilitate interpretation. The quantitative contribution of each biological score to atherogenesis across the dietary conditions was calculated from a multivariate predictive equation. Interaction graphs allowed us to report the cross talk among the biological functions in modulating atherosclerosis, as well as the differential responses of these functions to the various dietary fats.

\section{MATERIALS AND METHODS}

\section{Animals and Diets}

All of the experiments were approved by the referee for animal care at our institution and were conducted in accordance with the French Regulations for Animal Experimentation (Art. 19, Oct .1987, Ministry of Agriculture). All of the animal procedures conformed to the guidelines from Directive 2010/63/EU of the European Parliament on the protection of animals used for scientific purposes. Forty male Golden Syrian hamsters (9-wk-old, Janvier strain weighing 65-70 g) were purchased from the Janvier Breeding Center (Le Genest-St. Isle, France). Each hamster was individually caged and fed a commercial chow diet (25/18 standard diet from Mucedola, Settimo, Milan, Italy) for a 1-wk adaptation period. Eight hamsters per group were then fed experimental diets $(200 \mathrm{~g} / \mathrm{kg})$ for $12 \mathrm{wk}$. The various fats were: regular butter (RB); RB diluted to $50 \%$ (by weight) by a vegetable oil mixture made with $50 \%$ canola oil, $30 \%$ high oleic sunflower oil, and $20 \%$ sunflower oil (VRB); a low cholesterol butter diluted with the above plant oil mix (VLCB); a low cholesterol and a low saturated fatty acid butter (LCSB); and a plant oil mixture of 30\% canola oil and $70 \%$ palm oil (VM). Diet and water were available ad libitum. An anti-oxidant mixture (Oxy'Block; Naturex, Avignon, France), containing carnosic acid and carnosol (8\%) and soya tocopherols (35\%) completed to $100 \%$ with sunflower oil, was added at $300 \mathrm{ppm}$ to the LCSB fat. The dairy fats were provided by Corman. The compositions of the various fat diets are listed in Table 1.

\section{Blood Sampling and Chemical Assays}

The hamsters were anesthetized 5-8 h after their last meal by an intramuscular injection of Zoletil 50 at a dose of $250 \mathrm{mg} / \mathrm{kg}$. A cardiac puncture was used to collect 3-4 $\mathrm{ml}$ of blood, and plasma aliquots were stored at $-80^{\circ} \mathrm{C}$ until ready for analysis.

Plasma lipids were measured by enzymatic procedures using commercial kits (Biomerieux, Lyon, France), as follows: total cholesterol (RTU method) and triglycerides and phospholipids (PAP 150 method) (59).

HDL-cholesterol (HDL-Chol) was measured after dextran precipitation (Abcam ab65390 assay kit, Cambridge, UK), and the nonHDL-Chol (VLDL + LDL) was calculated by the difference between total cholesterol and HDL-Chol.

Two milliliters of whole blood were also collected in PAXgene blood RNA tubes (Qiagen, Courtaboeuf, France) and were stored at $-80^{\circ}$ for further quantitative PCR analysis of gene expression.

\section{Aorta Sampling}

For each hamster, the aorta was rinsed by perfusing cold saline from the left ventricle and was excised from the aortic arch up to the inferior iliac bifurcation. Whole aortic lipids were extracted and analyzed by gas liquid chromatography (LC), using a high-temperature nonpolar capillary column $(33,60)$. Whole aortic cholesteryl esters were considered to indicate early atherogenesis (fatty streaks) and were expressed as percentages of cholesteryl-esters of tissue phospholipids $(33,59)$. Such biochemical analysis was preferred over staining methods due to practical considerations and better accuracy than staining evaluations.

\section{Metabolomics}

Sample preparation. Urine samples were collected with a needle inserted directly into the bladder after anesthesia and were stored at $-80^{\circ}$ before processing, thus preventing any oxidation. After thawing, the samples were processed by centrifugation at $17,500 \mathrm{~g}$ for $10 \mathrm{~min}$ at $4^{\circ} \mathrm{C}$ and then were diluted with ultra-pure water $(1: 4$ $\mathrm{vol} / \mathrm{vol}$ ) and centrifuged again at $8,000 \mathrm{~g}$ for $5 \mathrm{~min}$. The supernatant was collected and kept at $-80^{\circ} \mathrm{C}$ until analysis.

Blood was collected from the left ventricle in heparinized tubes and left for no more than $1 \mathrm{~h}$ in a bed of ice. Plasma was separated by centrifugation $(2,000 \mathrm{~g} 10 \mathrm{~min})$ and was stored immediately at $-80^{\circ}$. Plasma was thawed before extraction. Metabolite extraction has been validated elsewhere $(31,46)$. Polar compounds in plasma were extracted by mixing $400 \mu \mathrm{l}$ of cold methanol with $200 \mu \mathrm{l}$ of plasma. After a minimum of $30 \mathrm{~min}$ of incubation at $-20^{\circ} \mathrm{C}$, the samples were thoroughly shaken and then centrifuged for $15 \mathrm{~min}$ at $11,000 \mathrm{rpm}$ and $4{ }^{\circ} \mathrm{C}$. The supernatants were transferred to fresh microtubes and centrifuged again for 15 min under the same conditions. The supernatants were transferred again into fresh tubes, dried under nitrogen flow, and stored at $-80^{\circ} \mathrm{C}$ before analysis. To assess the data quality, a blank sample (deionized water) and a pooled sample (a mixture of all of the samples) were extracted/diluted and analyzed repeatedly along with the sample series (49).

Liquid chromatography-mass spectrometry analysis. The samples were analyzed on an Agilent 1200 RRLC coupled to a Brüker microTOF ESI-hybrid quadrupole-time of flight mass spectrometer (Wissembourg, France). The LC conditions and mass spectrometry (MS) acquisition and data processing have already been fully described $(16,49)$ and are additionally reported in the Supplemental Material (Supplemental Material for this article is available online at the Journal website).

For the purpose of further metabolite identification, MS/MS analyses were performed on selected ions in the urine and plasma samples and completed by real standard analysis whenever possible. When identification was made with standard molecules, both retention times and MS/MS fragmentation spectra were used to confirm metabolite annotation. For MS/MS, the MRM parameters were set at 8.0 for isolation width, 2.0 for acquisition factor, and 10.0, 20.0, and $30.0 \mathrm{eV}$ for collision energies. Solutions of $1 \mathrm{mg} / \mathrm{ml}$ were prepared in different solvent mixtures according to each molecule's $\operatorname{LogP}$ value and were injected at a $10 \mu \mathrm{g} / \mathrm{ml}$ concentration. MS/MS spectra analyses and metabolite assignments are reported in the Supplemental Table S1.

\section{Gas-Chromatography Fatty Acid Analysis}

Five-hundred microliters of plasma and $100 \mu \mathrm{g}$ of liver tissues were extracted according to the methods of Bligh and Dyer (7) and of Folch et al. (12). Plasma lipid extracts were fractionated by thin-layer chromatography, and the fatty acid moieties of the fractionated lipid classes were transmethylated using boron trifluoride 5\% in methanol (Sigma-Aldrich, L'Isle d'Abeau Chesnes, France) (32). Fatty acids in total liver lipid extracts were transmethylated using acetyl-chloride in methanol, as described earlier (11). Fatty acid methyl esters were 
Table 1. Diet composition, including the fatty acids, cholesterol, phytosterol, and tocopherol contents of the lipid moiety

\begin{tabular}{|c|c|c|c|c|c|}
\hline Diet Components & $\mathrm{RB}$ & VRB* & $\mathrm{VLCB} \dagger$ & LCSB & $\mathrm{VM} \dagger$ \\
\hline \multicolumn{6}{|l|}{ Nutrients, g/100 g } \\
\hline DL-Methionine & 0.16 & 0.16 & 0.16 & 0.16 & 0.16 \\
\hline Starch & 39.84 & 39.84 & 39.84 & 39.84 & 39.84 \\
\hline Sucrose & 8 & 8 & 8 & 8 & 8 \\
\hline Casein & 22 & 22 & 22 & 22 & 22 \\
\hline Lipids & 20 & 20 & 20 & 20 & 20 \\
\hline Fiber & 5 & 5 & 5 & 5 & 5 \\
\hline Minerals & 4 & 4 & 4 & 4 & 4 \\
\hline Vitamins mix & 1 & 1 & 1 & 1 & 1 \\
\hline Energy, $\mathrm{MJ} / \mathrm{kg}$ & 19.5 & 19.5 & 19.5 & 19.5 & 19.5 \\
\hline \multicolumn{6}{|l|}{ Fatty acids, g/100 g } \\
\hline $\mathrm{C} 4: 0$ & 3.4 & 1.7 & 1.7 & 0.7 & 0 \\
\hline C6:0 & 2.2 & 1.1 & 1.1 & 0.8 & 0 \\
\hline C8:0 & 1.3 & 0.6 & 0.6 & 0.6 & 0 \\
\hline C10:0 & 2.6 & 1.3 & 1.4 & 1.8 & 0.1 \\
\hline $\mathrm{C} 10: 1$ & 0.2 & 0.1 & 0.1 & 0.2 & 0 \\
\hline $\mathrm{C} 12: 0$ & 3.2 & 1.6 & 1.7 & 2.3 & 0.2 \\
\hline $\mathrm{C} 14: 0$ & 10.8 & 5.3 & 5.4 & 6.8 & 0.8 \\
\hline C14:1 & 1.7 & 0.8 & 0.9 & 2.3 & 0 \\
\hline $\mathrm{C} 15: 0$ & 1.1 & 0.5 & 0.6 & 0.7 & 0.1 \\
\hline C16:0 & 30.2 & 17.2 & 17.1 & 17.6 & 32.4 \\
\hline $\mathrm{C} 16: 1(c+t)$ & 2.5 & 1.3 & 1.4 & 3.7 & 0.4 \\
\hline $\mathrm{C} 17: 0$ & 0.7 & 0.4 & 0.3 & 0.5 & 0.1 \\
\hline $\mathrm{C} 17: 1$ & 0.3 & 0.2 & 0.2 & 0.5 & 0 \\
\hline $\mathrm{C} 18: 0$ & 11.1 & 6.8 & 6.8 & 7.9 & 3.6 \\
\hline $\mathrm{C} 18: 1(c+t)$ & 23.9 & 42.6 & 42.3 & 43.7 & 45.5 \\
\hline C18:2n-6 & 2.4 & 14.0 & 13.9 & 4 & 12.7 \\
\hline CLA $(c+t)$ & 0.6 & 0.3 & 0.3 & 1.5 & 0 \\
\hline $\mathrm{C} 18: 3 n-3$ & 0.4 & 2.7 & 2.6 & 0.9 & 2.9 \\
\hline$n-6 / n-3$ & 5.6 & 5.2 & 5.3 & 4.7 & 4.4 \\
\hline Total saturated & 66.6 & 36.5 & 36.7 & 39.7 & 37.3 \\
\hline Total mono & 28.6 & 45 & 44.9 & 50.4 & 45.9 \\
\hline Total poly & 3.4 & 17 & 16.8 & 6.4 & 15.6 \\
\hline Polyunsaturated/saturated & 0.05 & 0.47 & 0.46 & 0.16 & 0.42 \\
\hline Unidentified & 1.4 & 1.5 & 1.6 & 3.5 & 1.2 \\
\hline Cholesterol $\mathrm{mg} / 100 \mathrm{~g}$ fat ( $\%$ in the diet) & $240(0.05)$ & $120(0.025)$ & $>26(0.005)$ & $20(0.004)$ & 0 \\
\hline Total phytosterols, ppm & 212 & 55,425 & 57,220 & 129 & 62,000 \\
\hline Stigmasterol & 6 & 25 & 24 & Not detected & 13 \\
\hline$\beta$-Sitosterol & 6 & 1,400 & 1,200 & 3 & 960 \\
\hline Campesterol & 200 & 54,000 & 56,000 & 126 & 61,000 \\
\hline Total tocopherol, $\mu \mathrm{g} / \mathrm{g}$ fat & 75.4 & 655.7 & 408.7 & 530.9 & 528.4 \\
\hline Alpha, \%total) & $37.7(50.0)$ & $516.6(78.8)$ & $308.6(75.5)$ & $51.7(9.7)$ & $316.9(60.0)$ \\
\hline Beta, \%total) & $16.0(21.2)$ & $14.9(2.3)$ & $9.9(2.4)$ & $17.0(3.2)$ & $10.0(1.9)$ \\
\hline Gamma, \%total & $19.7(26.1)$ & $123.2(18.8)$ & $89.4(21.9)$ & $372.8(70.2)$ & $194.3(36.8)$ \\
\hline Delta, \%total & $2.0(2.7)$ & $1.0(0.2)$ & $0.7(0.2)$ & $89.4(16.8)$ & $7.2(1.4)$ \\
\hline
\end{tabular}

$\mathrm{RB}$, regular butter; VRB, regular butter and 50\% vegetable oil mix 1; VLCB, low cholesterol butter and 50\% vegetable mix 1; LCSB, low cholesterol and low saturated fat butter; VM, vegetable mix 2. *Vegetable oil mix 1, made with 50\% canola oil, 30\% high oleic sunflower oil, $20 \%$ sunflower oil. $\dagger$ Vegetable oil mix 2, made with $30 \%$ canola oil and $70 \%$ palm oil.

analyzed by fast-GC (Perkin-Elmer, model Clarus 600, Les Ulis, France) (11).

\section{Quantitative PCR for Gene Expression Analysis}

Total RNA was isolated from liver samples using Nucleopin RNA II purification columns (Macherey-Nagel, Horedt, France) according to the suppliers' instructions. Blood RNA preserved in the PAXgene RNA tubes was extracted according to the instructions of the PAXGene RNA kit. The integrity of the RNA was assessed using an Agilent 2100 Bioanalyser; RNA samples scoring an RIN greater than 8 were considered of good quality and were used for further analysis. From $1.5 \mu \mathrm{g}$ of RNA extracted from liver samples or $0.4 \mu \mathrm{g}$ of RNA extracted from blood samples, cDNA was synthesized using Superscript-II Reverse Transcription kit (Invitrogen, Les Ulis, France) and was diluted in sterile water to final volumes of 340 and $100 \mu l$, respectively. Gene expression levels were determined using real-time PCR (on a Stratagene Mx 3005, La Jolla, CA) with SYBR Green as a fluorescent dye. $18 \mathrm{~S}$ was used as a reference gene because it showed stable expression for all of the diet groups (data not shown). All of the pairs of primers were previously validated (36), and the stability of the Tm products was verified after each run. All of the biological samples were run in duplicate. Gene expression was calculated using the mean normalized expression. The expression of 44 genes implicated in the metabolic control was examined in each hamster (35 in liver and 9 in whole blood; Supplemental Fig. S1).

\section{Statistics}

All partial least squares (PLS) models and analyses were performed using SIMCA P + 12 (Umetrics, Umea, Sweden). Interaction networks were calculated using Cytoscape (52), hierarchical analyses with Permutmatrix (8), receiver operator characteristic (ROC) curve and random forest analyses with Metaboanalyst (66), naïve Bayes analyses with Tanagra (48) (http://eric.univ-lyon2.fr/ ricco/tanagra/), univariate statistics with Statview (SAS Institute, Cary, NC), and partial correlations with the $\mathrm{R}$ package GeneNet. The statistical workflow in connection with the analytical analyses is shown is Fig. 1. The 
purpose was to select the most relevant variables relative to atherogenic status (step 1, PLS methods), to aggregate these variables into biological clusters according to statistical or ontological considerations (step 2), to evaluate the impact of the dietary treatment on each biological cluster (step 3), and to display the concerted action and the contribution of each biological cluster to the modulation of atherosclerosis (step 4).

\section{RESULTS}

\section{Diets and Atherogenic Status}

There were no differences in weight gain among the hamsters fed the various diets at the end of the nutritional trial (Table 2). The liver weights were slightly lower in the hamsters deprived of cholesterol in their diet (Table 2). Conversely, the atherogenic outcomes were dramatically affected by the lipid diets given to the hamsters (Fig. 2B). This atherogenic outcome was defined as the ratio of total cholesteryl-ester to total phospholipids in the aorta (see MATERIALS AND METHODS). In fact, the atherogenic status obtained with the experimental fat diets (VRB, VLCB, and LCSB with athero indexes of 4.47, 4.26 , and 3.35, respectively) were framed by the highest values found in the RB hamster group (athero index of 6.34) and the lowest values found in the VM hamster group (athero index of 1.86). It should be noted that all of the hamsters were atherosclerotic compared with hamsters fed a low-fat chow diet for the same period and used as "no-atherosclerotic" reference individuals (athero index of 0.99 , not shown). Atherogenicity was unrelated to the current intake of phytosterols in our study (Table 1 and Fig. 2, $A$ and $B$ ). Conversely, a lower atherogenic status was obtained by decreasing by half the total saturated fatty acids and cholesterol content in the diet (VRB diet), which was accomplished by diluting the RB with canola oil. Further lowering cholesterol intake to $80 \%$ (VLCB) by removing the cholesterol from dairy fat while keeping the other nutrients, comparable to the VRB diet did not significantly improve the atherogenic outcome. However, using a technological process to decrease both the saturated fatty acids and cholesterol (LCSB) similarly was more efficient in improving the atherogenic status $(-12.5 \% ; P<0.05$; Fig. $2 B)$.

\section{Biological Analyses and Atherogenic Status}

We also performed a multiplatform analysis of hamster biofluids and tissues to obtain better insights into the underlying mechanisms that might be associated with the severity of atherosclerosis. We performed untargeted metabolomics on urine and plasma samples, along with fatty acid analysis of plasma lipid classes and total liver lipids, analysis of total lipid classes in the whole aorta, analysis of the expression of 44 target genes in the liver and in whole blood involved in the metabolic control associated to atherogenic status (Supplemental Fig. S1), and analysis of conventional blood chemistry to assess the blood lipoprotein particles and lipid contents (Fig. 1). These analyses generated a set of 1,666 variables per hamster. Our statistical model retained 87 variables strongly

\section{Multiplatform data analysis $\times 40$ hamsters}
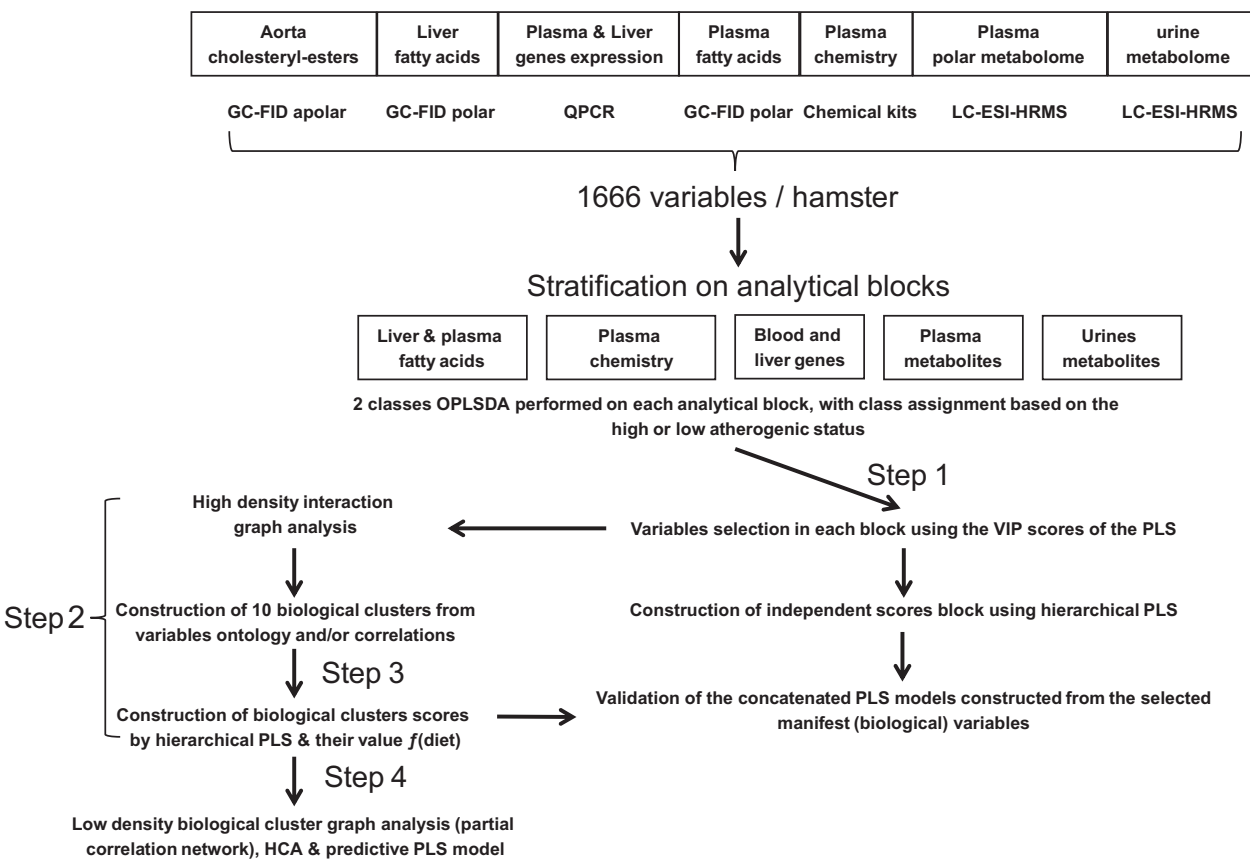

Fig. 1. Analytical and statistical workflow. To facilitate data processing and interpretation, the hamsters were stratified according to the median of their atherogenic index values (total cholesteryl-ester content relative to structural phospholipids in the whole aorta), into low or high atherogenic status groups. An orthogonal partial least square discriminant analysis (PLS-DA), using low or high atherogenic status as a class variable, was applied to each variable category (blocks), e.g., either liquid chromatography-mass spectrometry features in urine or in plasma, fatty acids both in liver and plasma, plasma biochemistry, or liver and blood gene expression. The combined model obtained after applying selected variables to individual variable categories was highly significant, with elevated explained variance of both observations and classes (Supplemental Table S3). From the initial 1,666 variables, this model, after curation for redundancies and artifacts, finally retained 87 variables significantly correlated with atherosclerosis (Supplemental Table S2). LC-ESI-HRMS, liquid chromatography-electrospray ionization-high resolution mass spectrometry; GC-FID, gas-chromatography flame ionization detection; HCA, hierarchical clustering analysis; QPCR, quantitative polymerase chain reaction, PLS; projection on latent structure; OPLS-DA, orthogonal projection on latent structure discriminant analysis. 
Table 2. Physiological outcomes of hamsters fed different diets after 12 wk of nutritional challenge

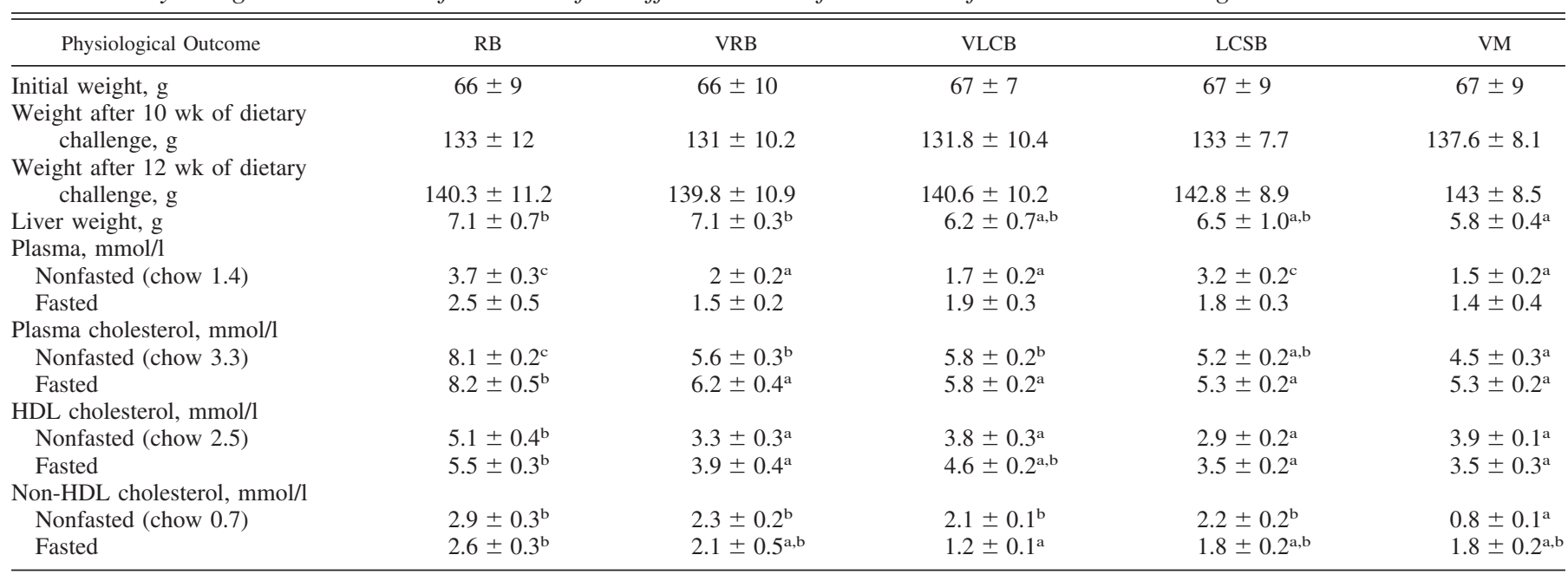

Values are means $\pm \mathrm{SD} ; n=8$ hamsters per group. Different letters on the same line indicate a statistical significance (Bonferroni-Dunn test). Chow values correspond to plasma values measured in 2 chow-fed hamsters for the same period.

associated with the atherogenic index, including some metabolomics features that remained unidentified (Supplemental Table S2).

\section{Statistical Modeling of Diological Clusters to Predict the Atherogenic Status}

To consider the complex dimensions of atherosclerosis, we visualized the relationships among the 87 variables that we found to be associated with the disease, using an interaction network. The network self-organized around the atherogenic index surrounded by different regions of variables. These regions of variables, in turn, defined 10 biological clusters, as described in Supplemental Fig. S2 and Supplemental Table S2.

To facilitate data interpretation while keeping all of the significant information, we modeled the 10 biological clusters into numerical biological scores as we already reported else-

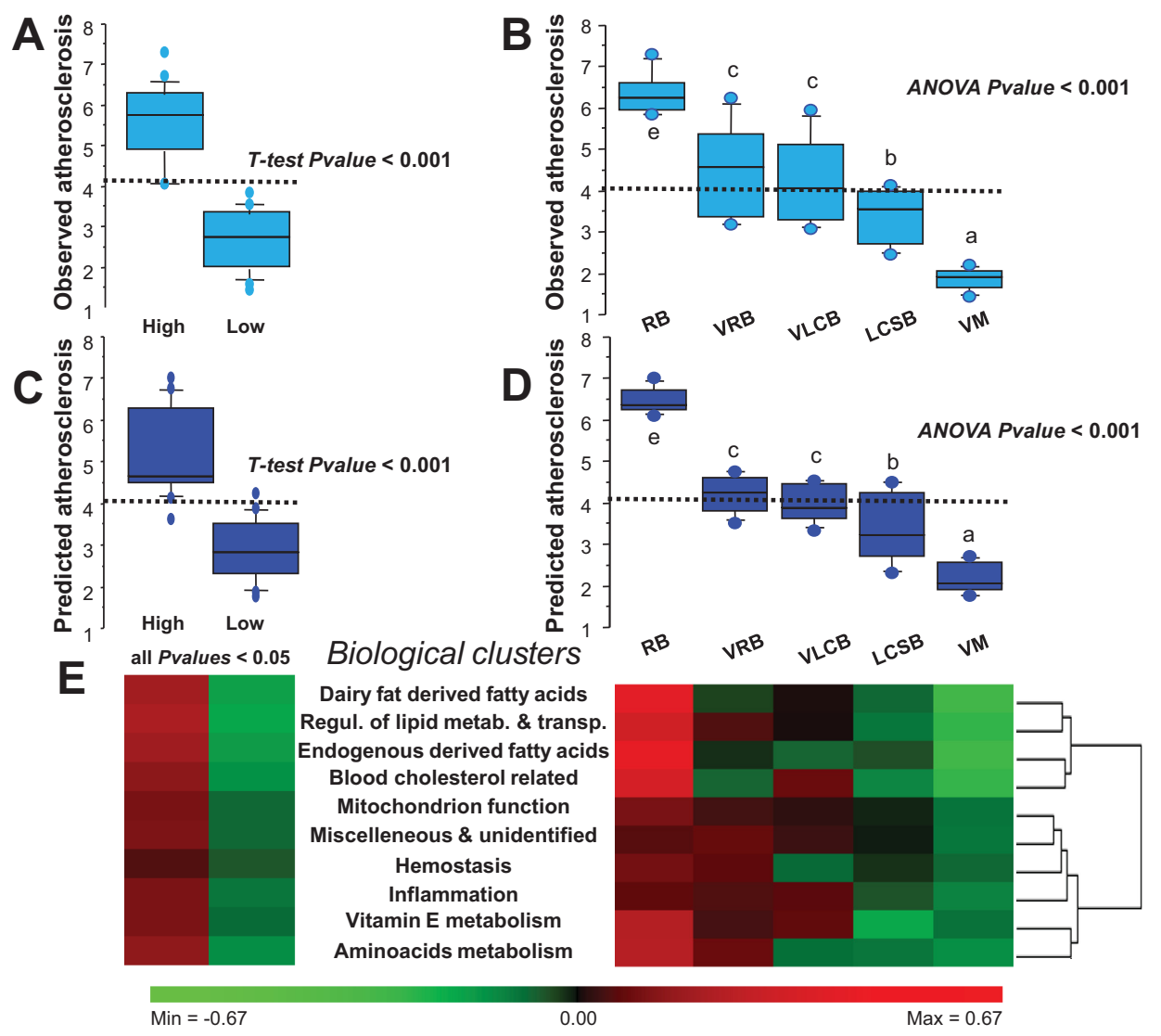

Fig. 2. Prediction of atherosclerosis with selected biological clusters. Observed atherosclerosis is expressed as a percentage of relative total cholesteryl-ester deposition in the aorta (to total phospholipids) in the hamsters with low or high atherogenic status $(A)$ or in the hamsters fed the various diets $(B)$. Corresponding predicted atherosclerosis is shown in $C$ and $D$. Predicted atherosclerosis is calculated from the PLS equation: pred athero $=0.108091 \times[$ dairy fat derived fatty acids] $+0.152669 \times$ [endogenous derived fatty acids] $-0.0340185 \times$ [mitochondrion function] $+0.173429 \times$ [regul. lipid metabol. transport] $-0.0751566 \times$ [vitamin E metabolism] $-0.153142 \times$ [hemostasis] $0.110269 \times$ [amino acid metabolism] + $0.110269 \times$ [blood cholesterol related] + $0.231903 \times$ [inflammation] $-0.00456344 \times$ [miscellaneous and unidentified] +4.06731 . $E$ : heat map of the quantitative contribution of each biological score to the atherogenic index. The results shown are from the hamsters with either low or high atherogenic status or in the hamsters fed the various diets. Red color indicates increased values, and green color indicates decreased values. See Table 3 for corresponding numerical values; $n=8$ hamsters per group. Dietary fat correspondence: $\mathrm{RB}$, regular butter; VRB, regular butter and $50 \%$ vegetable oil mix; VLCB, low cholesterol butter and $50 \%$ vegetable mix; LCSB, low cholesterol and saturated butter; VM, vegetable mix. Groups sharing a different lowercase letter $(\mathrm{a}, \mathrm{b}, \mathrm{c}, \mathrm{e})$ are significantly different $(P<0.05$, Tukey post hoc test after ANOVA). 
where (56), using a validated mathematical method (64) that is detailed in the supporting material. Each biological score can be observed as a multiplex variable computed from the original variables, defining the biological clusters. Such a strategy, using multi-omics data assembled into biological modules, was recently published when this article was under revision (63). With the use of the scores of the biological clusters, it was possible using the PLS algorithm to calculate a predictive equation of the atherogenic index for each hamster, as follows: predicted atherogenicity $=0.108091 \times$ [dairy fat derived fatty acids] $+0.152669 \times$ [endogenous derived fatty acids] $0.0340185 \times$ [mitochondrion function] $+0.173429 \times$ [regul. lipid metabol. trans.] $-0.0751566 \times$ [vitamin E metabolism] $0.153142 \times$ [hemostasis] $-0.110269 \times$ [amino acid metabolism $]+0.110269 \times$ [blood cholesterol related $]+0.231903 \times$ [inflammation] $-0.00456344 \times$ [miscellaneous and unidentified] +4.06731 . We previously applied this type of predictive equation to cardiovascular risk using gene single nucleotide polymorphism in humans (10). When considering a hamster with a low or a high atherogenic status, the predicted atherosclerosis level calculated from the equation above was similar to the observed atherosclerosis level (Fig. $2, A$ and $B$ ). The quantitative contribution of each biological cluster to the atherogenic index around this value and across the experimental diets thus could be easily calculated using the equation (Fig. $2 E$ and Table 3 for corresponding numerical values).

Integration of the Dietary Challenges, the Interplay Between the Biological Clusters, and the Atherogenic Status Using an Interaction Network

To gain further insight into the concerted interactions of the biological clusters to explain atherosclerosis, we calculated the partial correlations matrix among all of the cluster score values, together with the atherogenic index. The matrix was mapped as a network, and we focused on the most relevant interactions using $q$ values $\leq 0.10$ after false discovery rate filtering, to account for multiple comparisons (Fig. 3). This process yielded a very sparse network that was topologically divided in two parts, in which one could identify three important nodes having a hub position in the network, namely regulation of lipid transport and metabolism, inflammation, and amino acid metabolism. The node most statistically connected to atherosclerosis was regulation of lipid transport and metabolism, including SREBP1c as a regulating nuclear receptor and some of its target genes (ACC, SDC1, MTP, and LDL-r). The inflammation node connected the two parts of the network together. Amino acid metabolism also appeared as the main crossroad in the graph. In addition, we constructed a heat map to show how the individual biological cluster components were affected by the dietary treatments and which individual component was the most related to atherosclerosis (determined by their PLS-discriminant analysis-variable importance value).

\section{DISCUSSION}

The main goal of our study was to investigate the potential of various dairy fat design strategies to modulate early atherosclerosis. To examine the biological impact of the diets, we implemented a specific systems biology workflow that allowed for identifying and quantifying the contribution of biological sets associated with the disease and sensitive to dairy fat quality. For these purposes, we used the atherosclerosis-prone hamster model. This model shares similarity with humans in terms of cholesterol metabolism and has thus been frequently used in studies examining cholesterol-related cardiovascular diseases $(18,33,40,55,59,60)$.

\section{Justification of the Dietary Design and Atherogenic Impact of Diets}

Three test fats were evaluated, bounded by a reference dairy fat (regular butter) and a plant oil mixture, both of which were designed not to diverge too much from the test fats. The test fats were formulated to maintain the total saturated fat content at $<40 \%$ and the $n-6 / n-3$ polyunsaturated fatty acid ratio at $\sim 5$. It must be emphasized that our protocol was not designed, nor was it able, to examine the atherogenic activity of individual saturated fatty acids of various chain lengths but rather of total saturated fat. To determine possible interactions with dietary cholesterol, the fats were free of cholesterol or contained gradual amounts of cholesterol up to natural level (Table 1). It should be noted that the maximum amount of cholesterol in the test diets $(0.05 \%)$ was usually less than what has commonly been used in similar hamster studies $[0.12 \%$ (39) up to $3 \%$ (24)], making difficult strict comparisons with such studies. We chose these cholesterol amounts because they were similar to physiological conditions and we found them suitable for such a trial (33).

The three technologically modified dairy fats induced an intermediary atherosclerotic status (e.g., \%aorta cholesteryl ester to phospholipid ratio) compared with nonprocessed dairy fat (RB, higher atherosclerosis) or to the oil mixture (VM, lower atherosclerosis). The decholesterolized and desaturated dairy fat (LCSB) performed the best among the transformed dairy fats.

\section{Test-Fat-Specific Nutrient Content and Atherogenic Status}

To explain our findings further, we evaluated whether the various methods used to change the fatty acid and cholesterol content in our test fats also changed the content of other important "anti"-atherogenic nutrients, such as phytosterols or polyunsaturated (PUFA) C18 fatty acids, thus influencing the atherogenic status. If indeed the technological strategies that we used modified the phytosterol and PUFA content of the diets, these modifications were not obviously correlated with the atherogenic outcome. For instance, within a similar content of cholesterol, LCSB fat contained 400-fold less phytosterols and 3.4-fold less PUFA than VLCB but was still less atherogenic.

Overall, the lower atherogenic impact of the LCSB diet could seem paradoxical compared with the other dairy fat diets, particularly the VLCB diet, which is very close in composition but exhibited comparatively more atherogenic features. A difference in the amount of oxidative by-products in the diet cannot be argued to explain that difference. In fact, whereas we found lipoperoxidative fatty acids easily detectable under our LC-MS conditions (49), such compounds that could have occurred from oxidized diets were not found to be associated with atherosclerosis in the present study. One possibility is that the LCSB fat has higher levels of rumenic acid (2.5- to 5-fold 


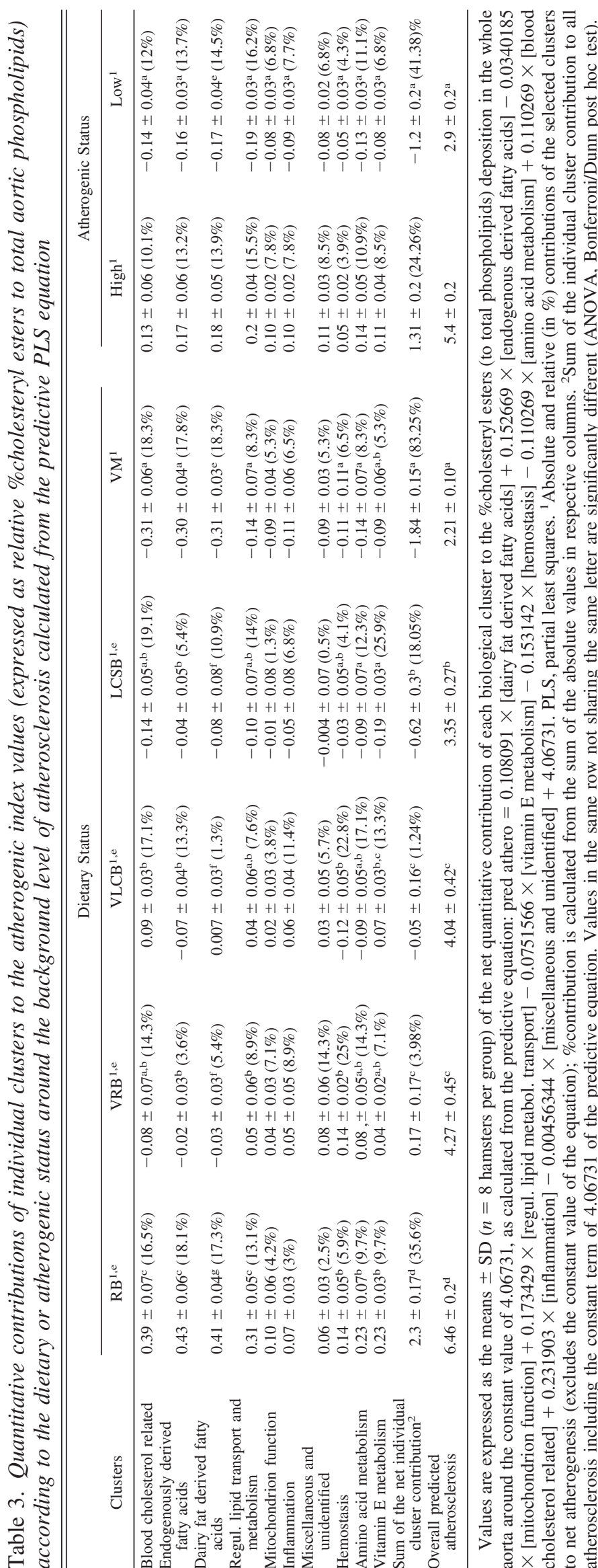

compared with other dairy fats), which is a conjugated linoleic acid (CLA), which we demonstrated to have strong antiatherogenic properties in milk fat-fed hamsters $(58,59)$. Such results with rumenic acid could also be extended to the apoE $\mathrm{E}^{-/-}$ atherogenic prone mouse model (3). Additionally, whereas total tocopherol contents matched across the diets (except for the standard butter diet RB), the LCSB diet was distinguished from the others by its higher content of the $\gamma$-tocopherol isomer. Indeed, it was specifically introduced by the supplier to prevent oxidation. Moreover, the nontargeted metabolomics analysis revealed that tocopherol urine metabolites, primarily $\gamma$-tocopherol with its main metabolite $\gamma$-CEHC $[2,7,8$ trimethyl-2-(beta-carboxyethyl)-6-hydroxychroman], were strongly and negatively associated with atherosclerosis, along with the $\beta$ - and $\delta$-isoforms. Strikingly, $\alpha$-CEHC originating from $\alpha$-tocopherol was not associated, although it was also detected in urine (not shown). Plasma $\gamma$-tocopherol was already found to be associated negatively with cholesterolinduced atherosclerosis in hamsters $(35,57,69)$. We also ascertained that this difference did not occur from a selective activation of the vitamin $\mathrm{E}$ degradation pathway at the gene expression level (Supplemental Fig. S3). The inverse association of the CEHC isoforms, and especially of $\gamma$-CEHC, with the atherogenic index in our study suggested that this vitamin E metabolite could be relevant for protection against cardiovascular disease even more than $\alpha$-tocopherol per se (17). Because there remain scanty data examining the protective cardiovascular action of $\gamma$-tocopherol in human, our findings at least support the conducting of clinical investigations in this area.

\section{Modeling into Scores the Biological Events Related to the Atherogenic Status Across the Dietary Treatments}

To investigate further how the atherogenic status was achieved across the dietary treatments, we determined which biological events were associated with the modulation of the disease by dairy fats. The heterogeneity in the origins of the 82 variables found to be associated with atherosclerosis provided a good illustration of the multifactorial basis of the disease that we intended to capture. To facilitate data interpretation and reporting, we aggregated the variables into 10 biological sets or clusters (Figs. 1 and 2 and Supplemental Table S2). When introduced into a PLS model, the 10 biological clusters described up to $81 \%$ of the atherogenic index variability $[\rho=$ $0.903, P=3.27 \times 10^{-7}$ after cross validated-ANOVA, ROCarea under the curve $(\mathrm{AUC})>0.90]$. In comparison, total fasted plasma cholesterol, a well-validated biomarker of atherosclerosis both in humans (25) and in the hyperlipidemic hamster (33), did not perform as well in our study $(\rho=0.58$, $P=0.00185$, ROC-AUC $=0.83$ ) nor did other fasted (nonHDL-C: $\rho=0.12, P=0.53$, ROC-AUC $=0.53$; non-HDL/ HDL-C ratio: $\rho=0.353, P=0.07$, ROC-AUC $=0.36$ ) or nonfasted plasma cholesterol values (total cholesterol: $\rho=$ $0.72, P<0.0001$, ROC-AUC $=0.84$; non-HDL-C: $\rho=0.66$, $P<0.0001$, ROC-AUC $=0.72$; non-HDL/HDL-C ratio: $\rho=$ $0.33, P=0.07$, ROC-AUC $=0.60)$. Indeed, assembling all of the fasted and non-fasted plasma cholesterol values into a PLS predictive atherogenic score did not perform as well as the 10 biological clusters $(\rho=0.76, P=0.000024$, $\mathrm{ROC}-\mathrm{AUC}=$ 0.84). 


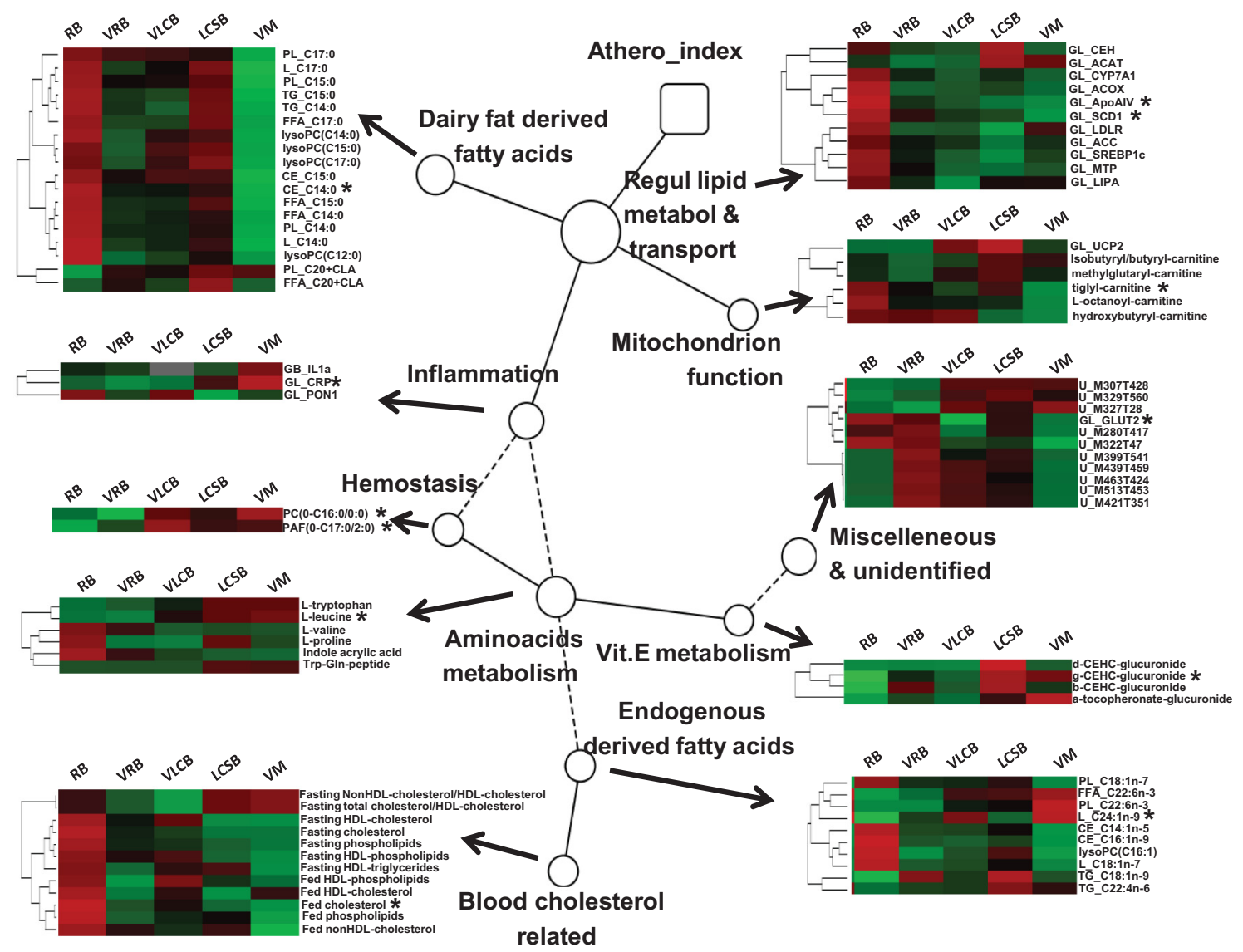

Fig. 3. Biological clusters low-density interaction network. The network was calculated from partial correlations. Each node depicts an individual biological cluster calculated from hierarchical PLS. The edges represent the partial correlation coefficients. The correlation network was built based on a correlation statistical significance of $q<0.10$ after the false discovery rate was determined; $n=8$ hamsters per group. The individual pathway components are displayed as a heat map, sorted according to hierarchical clustering (red box, relative increase; green box, relative decrease). *Indicates which individual components in the biological clusters weighed more in the biological score process and was more likely to predict atherosclerosis [greatest variable importance in projection (VIP) index of the PLS analysis].

Our quantitative approach, based on the biological clusters, also allowed for an interesting stratification of the relative contributions of complex biological events to the development of the disease. For instance, we found the four lipid-related bioclusters to be the main contributors to the atherogenic index, whether positively or negatively (Fig. 2 and Table 3 ). The amino acid and vitamin E metabolism bioclusters were secondary. For the latter, consistent with our finding of an inverse relationship between vitamin $\mathrm{E}$ metabolites and atherosclerosis, the vitamin $\mathrm{E}$ biocluster was the most quantitative contributor to atheroprotection in the LCSB-fed hamsters. All of these clusters were sensitive to the dietary fat quality, whereas the remainder of the other bioclusters were not (mitochondrion function, hemostasis, inflammation, miscellaneous, and unidentified). Beyond our study, such an approach could be relevant for choosing the most efficient strategy in situations of multitarget therapy and eventually in assessing the efficacy of therapy.

\section{Interplay Between the Biological Clusters Associated to the Atherogenic Atatus}

Interestingly, the regulation of lipid metabolism and transport biocluster, consisting exclusively of gene transcripts, was the main quantitative contributor to the atherogenic index
(16\%) among the hamsters of high or low atherogenic status, and it was especially greatly impacted by the RB diet (Table 3 ). It was also directly connected the atherogenic index (e.g., \% CE to PL ratio in the aorta) and to the remainder of the other bioclusters within the partial correlation interaction network (Fig. 3). This partial correlations network was efficient in detecting highly regulatory functions with hub positions (1), and it possesses a topology that could correspond to metabolic reactions (28). This biocluster thus appeared central to the concerted action of multiple biological events that led to atherosclerosis development, with an impact that was strongly correlated with dietary fat quality. Notable was that stearoyl CoA-desaturase I (SCD1) gene expression had greater influence in determining the score of that biocluster and was also correlated with many variables arising from other bioclusters (Supplemental Fig. S2). In contrast, SCD1 was also coregulated with other lipogenic genes regulated by the nuclear receptor SREBP1c, such as ACC, LDL-r, and MTP (Fig. 3), as well as other genes involved in lipid export from the liver (supplemental Fig. S2 and Fig. 3). Thus nonprocessed dairy fat (RB diet) fed in excess caused an overflow of circulating lipids that was aggravated by the triggering of endogenous lipogenesis, which ultimately led to more severe atherosclerosis. This process was illustrated well by the proximity with the other 
lipid biological clusters in hierarchical clustering analysis (blood cholesterol-related, endogenous-derived fatty acids, and dairy fat-derived fatty acids; Fig. 2E). Interestingly, such overflow was not observed with the transformed dairy fats and the plant oil mix control, despite the fat and energy intake being similar, underlying the qualitative impact of fats. Our finding also emphasized that the global regulatory role of SCD1 gene extended far beyond lipid metabolism, as demonstrated elsewhere (9), which was well illustrated in our correlation network (Supplemental Fig. S2).

\section{Data Integration with the Dietary Challenge and the Atherogenic Status}

Our results also stressed the relevance of decreasing both cholesterol levels (and/or availability) and saturated fatty acids to lower their atherogenic potential under conditions of high intake. In such inflationist intake conditions, the lipid overflow shifts the medium-chain saturated fatty acids lauric acid (C12:0) and myristic acid (C14:0) from neutral to atherogenic. Flooding the liver with fatty acids, such as the C14:0 compound, specifically inhibits cholesterol esterification and thus alters the distribution of excess cellular cholesterol in the liver between the storage pool of cholesteryl esters and the metabolically active, regulatory pool, as nicely explained some years ago (53). The link of such flooding with atherogenic status was well illustrated in our study by the association we found between the lipid species exhibiting dairy fat-derived fatty acids and atherosclerosis, particularly those esterifying myristic acid (Supplemental Fig. S2 and Fig. 3). In addition, the excess could also be responsible for some of the defects of the mitochondrion function biocluster, illustrated by the connection of this biocluster to the regulation of lipid transport and metabolism cluster in the interaction network. Hence, some of its components, i.e., hydroxybutyryl-, octanoyl-, and tiglyl-carnitines, associated with fatty acid oxidation and ketone body production in the mitochondria $(13,26)$, were higher in the more atherogenic hamsters. These metabolites likely reflected the defect in the management of lipid overflow discussed above through insufficient lipids. This downregulation of the UCP2 gene could also be viewed as a failure in mitochondrial fatty acid metabolism, contributing to atherosclerosis development (6). Additionally, butyryl/isobutyryl- and isovaleryl-, as well as tiglyl-carnitine, would reflect defects in branched-chain amino acids mitochondrion metabolism (leucine, isoleucine and valine) $(13,22,26)$. Finally, if we refer to LCSB hamsters, their acylcarnitine profile is close to that found in the highly atherogenic RB hamsters. However, the LCSB hamsters were poorly atherogenic. This proatherogenic feature was thus balanced by other regulations that could counteract such mitochondrial defects, exemplifying well how complex diseases arose when multiple disruptions remained unrepaired or were not offset and relying on a small set of biomarkers can be insufficient to conclude (34).

Additionally, beyond the impact on cholesterol metabolism, we also found that the dairy fat-derived fatty acids accumulated in lysoPCs in the blood, in correlation with the severity of atherosclerosis. In addition to reflecting dairy food intake (38), perhaps such lipid species esterifying medium-chain saturated fatty acids play a specific role in the progression of atherosclerosis, as reported with plasma long-chain lysoPCs $(15,44,54)$. This possibility certainly requires a precise evaluation. One of the mechanisms of lysoPC signaling occurs through interaction with either a uncharacterized receptor and/or the plateletactivating factor receptor in the monocytes, giving rise to arachidonic acid release and the synthesis of proinflammatory mediators (43). Along with lysoPCs, our untargeted metabolomics analysis also revealed platelet-activating factors (lysoPAF-O-C16:0 and PAF-O-C17:0/2:0) that were decreased in the low atherogenic status hamsters (Fig. 2E). As mentioned above, along with lysoPCs, such lipid messengers could also be involved in the progression of atherosclerosis. Nevertheless, not all PAF species exert the same biological response, and the one bearing the margaric alkyl (C17:0 alkyl) that we found has not yet been documented.

The amino acid metabolism biocluster formed a cross roads in the interaction network, underlying its significant importance in metabolic regulation, especially in relating vitE metabolism to inflammation. This statistical connection could illustrate the importance of unappreciated interplays between metabolites and/or biological clusters in the development of a complex disease such as atherosclerosis. Our results were also consistent with findings in humans relating branched-chain amino acids (amino acids biocluster) and their acylcarnitine products (mitochondrion function biocluster) to arterial disease (5), although the significance of the association could be different from that in our study.

We found within the inflammation biocluster that IL1a and CRP genes expression was upregulated in the least atherogenic high plant oil fed hamsters (VM) compared with the other dairy fat fed hamsters and the antioxidant PON1 was downregulated. This finding could seem paradoxical because such a profile is clearly proatherogenic. However, dairy fat intake has also frequently been associated with improvement of the inflammatory and oxidative status in obese subjects $(27,68)$, which could be consistent with our observations.

In conclusion, we found that, under conditions of atherogenic intake, the impact of dairy fats on the disease (RB diet) could be dramatically reduced by technological processing, such as by decreasing cholesterol and total saturated fatty acids (VRB, VLCB, and LCSB diets) and, further, by augmenting the level of antiatherogenic nutrients, such as CLA (59) and more likely $\gamma$-tocopherol (LCSB diet) (69). We thus additionally demonstrated that dairy fats cannot be considered monolithically with regard to cardiovascular diseases and that quality improvements with regard to this outcome could be achieved. For instance, marketing products including dairy fats, such as the LCSB, could provide health benefits for consumers or at least could help in the setting of a prudent diet. Very recently, such a strategy, using a canola oil-modified butter as part of a Mediterranean diet, was found to be quite effective for improving blood lipids in a randomized, controlled study of overweight individuals (4).

In our context, the atherogenic impact of individual saturated fatty acids could not be formally revealed, and PUFA levels did not seem to exert obvious effects. Of course, the hyperlipidemic hamster does not represent the general features of atherosclerosis and restricted our conclusions to these specific settings. Other animal models with genetically induced atherosclerosis could yield complementary information with regard to the background biological events underlying the disease outcome.

When comparing the various biological components related to our atherosclerosis conditions, it was possible to distinguish factors that were sensitive to dairy fat composition from those 
that were not or that were less sensitive to dairy fats. This distinction is important to know when addressing complex multifactorial diseases to choose the most appropriate and efficient dietary intervention strategy. From this perspective, we believe that the integrated analysis we performed, which allowed for the calculation of a predictive equation consisting of biological clusters, could be a powerful strategy for targeting the most relevant factors. For instance, we identified the cluster regulation of lipid metabolism and transport, including $\mathrm{SCD} 1$, as the main driver of the atherogenic status across our dietary settings, along with other biological events. The accuracy of our analysis could be further improved by even wider coverage of the "omics" dimensions. A very recent human study, using a similar multi-omics biological module concept applied in the context of cardiometabolic risk, was published when our article was in revision (63). This study and ours were the first examples, to our knowledge, that allowed for the precise quantitative contribution of various biological sets to a defined disease outcome. They illustrated how such tools could be constructed to identify, quantify, and stratify specifically the contributions of various components to the development of a complex disease such as atherosclerosis. This ability could be advantageous in situations of growing multimorbidity (2). Therefore, we consider our results to be very encouraging for the identification and validation of multiplex predictors, instead of single predictors of cardiovascular disease risk (50).

\section{GRANTS}

This work was partly supported by Corman.

\section{DISCLOSURES}

D. Dalemans is employed by Corman, which provided the transformed dairy fats and partially financed the study.

\section{AUTHOR CONTRIBUTIONS}

Author contributions: J.-C.M., D.D., and B.D. conception and design of research; J.-C.M., A.B., C.G., R.B., P.S., and J.-F.L. analyzed data; J.-C.M., M.-C.A., and B.D. interpreted results of experiments; J.-C.M. prepared figures; J.-C.M. and B.D. drafted manuscript; J.-C.M. and B.D. edited and revised manuscript; J.-C.M. and M.-C.A. approved final version of manuscript; A.B., C.G., R.B., P.S., A.S., and D.G. performed experiments.

\section{REFERENCES}

1. Allen JD, Xie Y, Chen M, Girard L, Xiao G. Comparing statistical methods for constructing large scale gene networks. PLoS One 7: e29348, 2012.

2. Anderson G, Horvath $\mathbf{J}$. The growing burden of chronic disease in America. Public Health Rep 119: 263-270, 2004.

3. Arbones-Mainar JM, Navarro MA, Guzman MA, Arnal C, Surra JC, Acin S, Carnicer R, Osada J, Roche HM. Selective effect of conjugated linoleic acid isomers on atherosclerotic lesion development in apolipoprotein E knockout mice. Atherosclerosis 189: 318-327, 2006.

4. Austel A, Ranke C, Wagner N, Gorge J, Ellrott T. Weight loss with a modified Mediterranean-type diet using fat modification: a randomized controlled trial. Eur J Clin Nutr 2015 Feb 18 [Epub ahead of print].

5. Bhattacharya S, Granger CB, Craig D, Haynes C, Bain J, Stevens RD, Hauser ER, Newgard CB, Kraus WE, Newby LK, Shah SH. Validation of the association between a branched chain amino acid metabolite profile and extremes of coronary artery disease in patients referred for cardiac catheterization. Atherosclerosis 232: 191-196, 2014.

6. Blanc J, Alves-Guerra MC, Esposito B, Rousset S, Gourdy P, Ricquier D, Tedgui A, Miroux B, Mallat Z. Protective role of uncoupling protein 2 in atherosclerosis. Circulation 107: 388-390, 2003.

7. Bligh EG, Dyer WJ. A rapid method of total lipid extraction and purification. Can J Biochem Physiol 37: 911-917, 1959.
8. Caraux G, Pinloche S. Permutmatrix: a graphical environment to arrange gene expression profiles in optimal linear order. Bioinformatics 21: 1280 1281, 2005.

9. Castro C, Sar F, Shaw WR, Mishima M, Miska EA, Griffin JL. A metabolomic strategy defines the regulation of lipid content and global metabolism by Delta9 desaturases in Caenorhabditis elegans. BMC Genomics 13: 36, 2012.

10. Desmarchelier C, Martin JC, Planells R, Gastaldi M, Nowicki M, Goncalves A, Valero R, Lairon D, Borel P. The postprandial chylomicron triacylglycerol response to dietary fat in healthy male adults is significantly explained by a combination of single nucleotide polymorphisms in genes involved in triacylglycerol metabolism. J Clin Endocrinol Metab 99: E484-488, 2014.

11. Du Q, Martin JC, Agnani G, Pages N, Leruyet P, Carayon P, Delplanque B. Dairy fat blends high in alpha-linolenic acid are superior to n-3 fatty-acid-enriched palm oil blends for increasing DHA levels in the brains of young rats. J Nutr Biochem 23: 1573-1582, 2012.

12. Folch J, Lees M, Stanley GH. A simple method for the isolation and purification of total lipids from animal tissues. J Biol Chem 226: 497-509, 1957.

13. Fukao T, Mitchell G, Sass JO, Hori T, Orii K, Aoyama Y. Ketone body metabolism and its defects. J Inherit Metab Dis 37: 541-551, 2014.

14. German JB, Gibson RA, Krauss RM, Nestel P, Lamarche B, van Staveren WA, Steijns JM, de Groot LC, Lock AL, Destaillats F. A reappraisal of the impact of dairy foods and milk fat on cardiovascular disease risk. Eur J Nutr 2009.

15. Goncalves I, Edsfeldt A, Ko NY, Grufman H, Berg K, Bjorkbacka H, Nitulescu M, Persson A, Nilsson M, Prehn C, Adamski J, Nilsson J. Evidence supporting a key role of Lp-PLA2-generated lysophosphatidylcholine in human atherosclerotic plaque inflammation. Arterioscler Thromb Vasc Biol 32: 1505-1512, 2012.

16. Grison S, Fave G, Maillot M, Manens L, Delissen O, Blanchardon E, Banzet N, Defoort C, Bott R, Dublineau I, Aigueperse J, Gourmelon P, Martin JC, Souidi M. Metabolomics identifies a biological response to chronic low-dose natural uranium contamination in urine samples. Metabolomics 9: 1168-1180, 2013.

17. Hodis HN, Mack WJ, LaBree L, Mahrer PR, Sevanian A, Liu CR, Liu CH, Hwang J, Selzer RH, Azen SP. Alpha-tocopherol supplementation in healthy individuals reduces low-density lipoprotein oxidation but not atherosclerosis: the Vitamin E Atherosclerosis Prevention Study (VEAPS). Circulation 106: 1453-1459, 2002.

18. Horton TJ, Drougas H, Brachey A, Reed GW, Peters GW, Hill JO. Fat and carbohydrate overfeeding in humans: different effects on energy storage. Am J Clin Nutr 62: 19-29, 1995.

19. Hu FB, Stampfer MJ, Manson JE, Ascherio A, Colditz GA, Speizer FE, Hennekens CH, Willett WC. Dietary saturated fats and their food sources in relation to the risk of coronary heart disease in women. Am J Clin Nutr 70: 1001-1008, 1999.

20. Huth PJ, Park KM. Influence of dairy product and milk fat consumption on cardiovascular disease risk: a review of the evidence. Adv Nutr 3: 266-285, 2012.

21. Jacques H, Gascon A, Arul J, Boudreau A, Lavigne C, Bergeron J. Modified milk fat reduces plasma triacylglycerol concentrations in normolipidemic men compared with regular milk fat and nonhydrogenated margarine. Am J Clin Nutr 70: 983-991, 1999.

22. Jeter CB, Hergenroeder GW, Ward NH, Moore AN 3rd, Dash PK. Human mild traumatic brain injury decreases circulating branched-chain amino acids and their metabolite levels. J Neurotrauma 30: 671-679, 2013.

23. Jove M, Ayala V, Ramirez-Nunez O, Serrano JC, Cassanye A, Arola L, Caimari A, Del Bas JM, Crescenti A, Pamplona R, Portero-Otin M. Lipidomic and metabolomic analyses reveal potential plasma biomarkers of early atheromatous plaque formation in hamsters. Cardiovasc Res 97: 642-652, 2013.

24. Kahlon TS, Chow FI, Irving DW, Sayre RN. Cholesterol response and fatty streak formation in hamsters fed two levels of saturated fat and various levels of cholesterol. Nutr Res 17: 1693-1707, 1997.

25. Keys A, Menotti A, Karvonen MJ, Aravanis C, Blackburn H, Buzina R, Djordjevic BS, Dontas AS, Fidanza F, Keys MH, Kromhout D, Nedeljkovic S, Punsar S, Seccareccia F, Toshima H. The diet and 15-year death rate in the seven countries study. Am J Epidemiol 124: 903-915, 1986.

26. Kompare M, Rizzo WB. Mitochondrial fatty-acid oxidation disorders. Semin Pediatr Neurol 15: 140-149, 2008. 
27. Kratz M, Baars T, Guyenet S. The relationship between high-fat dairy consumption and obesity, cardiovascular, and metabolic disease. Eur J Nutr 52: 1-24, 2013.

28. Krumsiek J, Suhre K, Illig T, Adamski J, Theis FJ. Gaussian graphical modeling reconstructs pathway reactions from high-throughput metabolomics data. BMC Syst Biol 5: 21, 2011.

29. Legrand P, Morise A, Kalonji E. Update of French nutritional recommendations for fatty acids. World Rev Nutr Diet 102: 137-143, 2011

30. Markey O, Vasilopoulou D, Givens DI, Lovegrove JA. Dairy and cardiovascular health: friend or foe? Nutr Bull 39: 161-171, 2014.

31. Martin JC, Maillot M, Mazerolles G, Verdu A, Lyan B, Migne C, Defoort C, Canlet C, Junot C, Guillou C, Manach C, Jabob D, Jouan-Rimbaud Bouveresse D, Paris E, Pujos-Guillot E, Jourdan F, Giacomoni F, Courant F, Favé G, Le Gall G, Chassaigne H, Tabet JC, Martin JF, Antignac JP, Shintu L, Defernez M, Philo M, AlexandreGouaubau MC, Amiot-Carlin MJ, Bossis M., Triba MN, Stojilkovic N, Banzet N, Molinié R, Bott R, Goulitquer S, Caldarelli S, Rutledge DN. Can we trust untargeted metabolomics? Results of the metabo-ring initiative, a large-scale, multi-instrument inter-laboratory study. Metabolomics 11: 807-821, 2015.

32. Martin JC, Bougnoux P, Antoine JM, Lanson M, Couet C. Triacylglycerol structure of human colostrum and mature milk. Lipids 28: 637-643, 1993.

33. Martin JC, Canlet C, Delplanque B, Agnani G, Lairon D, Gottardi G, Bencharif K, Gripois D, Thaminy A, Paris A. (1)H NMR metabonomics can differentiate the early atherogenic effect of dairy products in hyperlipidemic hamsters. Atherosclerosis 206: 127-133, 2009.

34. Mensink RP, Aro A, Den Hond E, German JB, Griffin BA, ten Meer HU, Mutanen M, Pannemans D, Stahl W. PASSCLAIM-diet-related cardiovascular disease. Eur J Nutr 42, Suppl 1: I6-27, 2003.

35. Mitchell PL, McLeod RS. Conjugated linoleic acid and atherosclerosis: studies in animal models. Biochem Cell Biol 86: 293-301, 2008.

36. Navarro V, Portillo MP, Margotat A, Landrier JF, Macarulla MT, Lairon D, Martin JC. A multi-gene analysis strategy identifies metabolic pathways targeted by trans-10, cis-12-conjugated linoleic acid in the liver of hamsters. Br J Nutr 102: 537-545, 2009.

37. Nestel PJ. Effects of dairy fats within different foods on plasma lipids. $J$ Am Coll Nutr 27: 735S-740S, 2008.

38. Nestel PJ, Straznicky N, Mellett NA, Wong G, De Souza DP, Tull DL, Barlow CK, Grima MT, Meikle PJ. Specific plasma lipid classes and phospholipid fatty acids indicative of dairy food consumption associate with insulin sensitivity. Am J Clin Nutr 99: 46-53, 2014

39. Nicolosi RJ, Wilson TA, Rogers E, Kritchevsky D. Effects of specific fatty acids (8:0, 14:0, cis-18:1, trans-18:1) on plasma lipoproteins, early atherogenetic potential, and LDL oxidative properties in the hamster. $J$ Lipid Res 39: 1972-1980, 1998

40. Nistor A, Bulla A, Filip DA, Radu A. The hyperlipidemic hamster as model of experimental atherosclerosis. Atherosclerosis 68: 159-173, 1987.

41. Noakes M, Nestel PJ, Clifton PM. Modifying the fatty acid profile of dairy products through feedlot technology lowers plasma cholesterol of humans consuming the products. Am J Clin Nutr 63: 42-46, 1996.

42. O'Sullivan TA, Hafekost K, Mitrou F, Lawrence D. Food sources of saturated fat and the association with mortality: a meta-analysis. Am J Public Health 103: e31-42, 2013

43. Oestvang J, Anthonsen MW, Johansen B. LysoPC and PAF trigger arachidonic acid release by divergent signaling mechanisms in monocytes. J Lipids 2011: 532145, 2011.

44. Ou ZJ, Li L, Liao XL, Wang YM, Hu XX, Zhang QL, Wang ZP, Yu H, Zhang X, Hu P, Xu YQ, Liang QL, Ou JS, Luo G. Apolipoprotein A-I mimetic peptide inhibits atherosclerosis by altering plasma metabolites in hypercholesterolemia. Am J Physiol Endocrinol Metab 303: E683-E694, 2012.

45. Pellizzon M, Ana JS, Buison E, Martin J, Buison A, Jen KL. Effect of a modified milk fat and calcium in purified diets on cholesterol metabolism in hamsters. Lipids 39: 441-448, 2004.

46. Pereira H, Martin JF, Joly C, Sébédio JL, Pujos-Guillot E. Development and validation of a UPLC/MS method for a nutritional metabolomic study of human plasma. Metabolomics 6: 207-218, 2010.

47. Puska P. Fat and heart disease: yes we can make a change-the case of North Karelia (Finland). Ann Nutr Metab 54, Suppl 1: 33-38, 2009.

48. Rakotomalala R. TANAGRA: un logiciel gratuit pour l'enseignement et la recherche. Actes de EGC'2005 2: 697-702, 2005.

49. Respondek F, Gérard P, Bossis M, Boschat L, Bruneau A, Rabot S, Wagner A, Martin JC. Short-chain fructo-oligosaccharides modulate intestinal microbiota and metabolic parameters of human microbiotaassociated diet induced obesity mice. PlosOne 8: 1-12, 2013.
50. Roberts LD, Gerszten RE. Toward new biomarkers of cardiometabolic diseases. Cell Metab 18: 43-50, 2013.

51. Seidel C, Deufel T, Jahreis G. Effects of fat-modified dairy products on blood lipids in humans in comparison with other fats. Ann Nutr Metab 49: 42-48, 2005

52. Shannon P, Markiel A, Ozier O, Baliga NS, Wang JT, Ramage D, Amin N, Schwikowski B, Ideker T. Cytoscape: a software environment for integrated models of biomolecular interaction networks. Genome Res 13: $2498-2504,2003$

53. Spady DK, Woolett LA, Dietschy JM. Regulation of plasma LDLcholesterol levels by dietary cholesterol and fatty acids. Annu Rev Nutr 13: 355-381, 1993

54. Tan M, Hao F, Xu X, Chisolm GM, Cui MZ. Lysophosphatidylcholine activates a novel PKD2-mediated signaling pathway that controls monocyte migration. Arterioscler Thromb Vasc Biol 29: 1376-1382, 2009.

55. Terpstra AHM, Holmes JC, Nicolosi RJ. The hypocholesterolemic effect of dietary soybean protein vs. casein in hamsters fed cholesterol-free or cholesterol-enriched semipurified diets. J Nutr 121: 944-947, 1991.

56. Thabuis C, Destaillats F, Lambert D, Muccioli GG, Maillot M, Harach T, Tissot-Favre D, Martin JC. Lipid transport function is the main target of oral oleylethanolamide to reduce adiposity in high-fat fed mice. J Lipid Res 1373-1382, 2011.

57. Toomey S, Roche H, Fitzgerald D, Belton O. Regression of preestablished atherosclerosis in the apoE-/- mouse by conjugated linoleic acid. Biochem Soc Trans 31: 1075-1079, 2003.

58. Valeille K, Ferezou J, Amsler G, Quignard-Boulange A, Parquet M, Gripois D, Dorovska-Taran V, Martin JC. A cis9,trans11-conjugated linoleic acid rich-oil reduces the outcome of atherogenic process in hyperlipidemic hamster. Am J Physiol Heart Circ Physiol 289: H652H659, 2005.

59. Valeille K, Ferezou J, Parquet M, Amsler G, Gripois D, QuignardBoulange A, Martin JC. The natural concentration of the conjugated linoleic acid, cis-9,trans-11, in milk fat has antiatherogenic effects in hyperlipidemic hamsters. J Nutr 136: 1305-1310, 2006.

60. Valeille K, Gripois D, Blouquit MF, Souidi M, Riottot M, Bouthegourd JC, Serougne C, Martin JC. Lipid atherogenic risk markers can be more favourably influenced by the cis-9,trans-11-octadecadienoate isomer than a conjugated linoleic acid mixture or fish oil in hamsters. Br J Nutr 91: 191-199, 2004.

61. Vandevijvere S, De Vriese S, Huybrechts I, Moreau M, Temme E, De Henauw S, De Backer G, Kornitzer M, Leveque A, Van Oyen H. The gap between food-based dietary guidelines and usual food consumption in Belgium, 2004. Public Health Nutr 12: 423-431, 2009.

62. Vartiainen E, Laatikainen T, Peltonen M, Juolevi A, Mannisto S, Sundvall J, Jousilahti P, Salomaa V, Valsta L, Puska P. Thirty-fiveyear trends in cardiovascular risk factors in Finland. Int J Epidemiol 39: 504-518, 2010.

63. Wahl S, Vogt S, Stuckler F, Krumsiek J, Bartel J, Kacprowski T, Schramm K, Carstensen M, Rathmann W, Roden M, Jourdan C, Kangas AJ, Soininen P, Ala-Korpela M, Nothlings U, Boeing H, Theis FJ, Meisinger C, Waldenberger M, Suhre K, Homuth G, Gieger C, Kastenmuller G, Illig T, Linseisen J, Peters A, Prokisch H, Herder C, Thorand B, Grallert H. Multi-omic signature of body weight change: results from a population-based cohort study. BMC Med 13: 48, 2015.

64. Wold S, Kettaneh N, Tjessem K. Hierarchical multiblock PLS and PC models for easier model interpretation and as an alternative to variable selection. J Chemometrics 10: 463-482, 1996.

65. Wolff E, Vergnes MF, Portugal H, Defoort C, Amiot-Carlin MJ, Lairon D, Nicolay A. Cholesterol-absorber status modifies the LDL cholesterol-lowering effect of a Mediterranean-type diet in adults with moderate cardiovascular risk factors. J Nutr 141: 1791-1798, 2011.

66. Xia J, Psychogios N, Young N, Wishart DS. MetaboAnalyst: a web server for metabolomic data analysis and interpretation. Nucl Acids Res 37: W652-660, 2009.

67. Yu-Poth S, Zhao G, Etherton T, Naglak M, Jonnalagadda S, Kris Etherton PM. Effects of the National Cholesterol Education Program's Step I and Step II dietary intervention programs on cardiovascular disease risk factors: a meta-analysis. Am J Clin Nutr 69: 632-646, 1999.

68. Zemel MB, Sun X, Sobhani T, Wilson B. Effects of dairy compared with soy on oxidative and inflammatory stress in overweight and obese subjects. Am J Clin Nutr 91: 16-22, 2010

69. Zha W, AJ, Wang G, Yan B, Gu S, Zhu X, Hao H, Huang Q, Sun J, Zhang Y, Cao B, Ren H. Metabonomic characterization of early atherosclerosis in hamsters with induced cholesterol. Biomarkers 14: 372-380, 2009 